\author{
Thomas Schertler \\ Hans Scheffel \\ Thomas Frauenfelder \\ Lotus Desbiolles \\ Sebastian Leschka \\ Paul Stolzmann \\ Burkhardt Seifert \\ Thomas G. Flohr \\ Borut Marincek \\ Hatem Alkadhi
}

\section{Dual-source computed tomography in patients with acute chest pain: feasibility and image quality}

Received: 22 April 2007

Revised: 12 June 2007

Accepted: 6 July 2007

Published online: 13 September 2007

(C) Springer-Verlag 2007

T. Schertler · H. Scheffel

T. Frauenfelder · L. Desbiolles ·

S. Leschka · P. Stolzmann ·

B. Marincek - H. Alkadhi $(\square)$

Department of Medical Radiology,

Institute of Diagnostic Radiology,

University Hospital Zurich,

Raemistrasse 100CH-8091,

Zurich, Switzerland

e-mail: hatem.alkadhi@usz.ch

B. Seifert

Department of Biostatistics,

University of Zurich,

Zurich, Switzerland

T. G. Flohr

Siemens Medical Solutions,

Computed Tomography CTE PA,

Forchheim, Germany

\begin{abstract}
The aim of this study was to determine the feasibility and image quality of dual-source computed tomography angiography (DSCTA) in patients with acute chest pain for the assessment of the lung, thoracic aorta, and for pulmonary and coronary arteries. Sixty consecutive patients (32 female, 28 male, mean age $58.1 \pm$ 16.3 years) with acute chest pain underwent contrast-enhanced electrocardiography-gated DSCTA without prior beta-blocker administration. Vessel attenuation of different thoracic vascular territories was measured, and image quality was semi-quantitatively analyzed by two independent readers. Image quality of the thoracic aorta was diagnostic in all 60 patients, image quality of pulmonary arteries was diagnostic in 59, and image quality of coronary arteries was di-
\end{abstract}

agnostic in 58 patients. Pairwise intraindividual comparisons of attenuation values were small and ranged between $1 \pm 6 \mathrm{HU}$ comparing right and left coronary artery and $56 \pm 9 \mathrm{HU}$ comparing the pulmonary trunk and left ventricle. Mean attenuation was $291 \pm 65 \mathrm{HU}$ in the ascending aorta, $334 \pm 93 \mathrm{HU}$ in the pulmonary trunk, and $285 \pm 66 \mathrm{HU}$ and $268 \pm 67 \mathrm{HU}$ in the right and left coronary artery, respectively. DSCTA is feasible and provides diagnostic image quality of the thoracic aorta, pulmonary and coronary arteries in patients with acute chest pain.

Keywords Acute chest pain . Dual-source CT .

Coronary angiography

\section{Introduction}

Acute chest pain represents one of the most difficult diagnostic challenges in emergency medicine. Chest pain history alone often cannot identify a group of patients who could be treated without further diagnostic testing [1], and triage decisions based on initial cardiac enzyme levels [2] and electrocardiography (ECG) [3] are often insufficient. Imaging may improve patient triage by decreasing delay in diagnosis and treatment and thus morbidity and mortality $[4,5]$. Most studies [6-8] have focused on patients being suspected of having acute coronary syndrome, but did not include additional differential diagnoses such as aortic dissection or pulmonary embolism, conditions that may clinically mimic coronary syndromes [9].
Multi-detector row computed tomography (CT) angiography is widely accepted and routinely used as a primary tool in the emergency assessment of pulmonary embolism [10] and aortic dissection [11]. In addition, 64-slice CT coronary angiography has demonstrated the capability to diagnose and to rule-out coronary artery disease (CAD) $[12,13]$. Some recent studies have shown that ECG-gated multi-detector row CT is logistically feasible [14] and yields promising results as a modality for evaluating chest pain patients with cardiac and non-cardiac disease in the emergency setting $[15,16]$. Even though using 64-slice CT scanner technology, however, patients with elevated heart rates require medical heart rate control prior to $\mathrm{CT}$ coronary angiography by administering beta-blockers and/ or benzodiazepines [8, 13, 17]. 
Dual-source CT (DSCT) represents the most recent scanner technology and is characterized by two tubes and two detectors that are mounted in orthogonal orientation onto the gantry. As compared to 64-slice CT, this scanner configuration maintains a consistently high spatial resolution while enabling ECG-gated imaging with an increased temporal resolution of $83 \mathrm{~ms}$ [18]. First studies have shown robust results of DSCT angiography (DSCTA) regarding image quality of cardiac structures even at high heart rates [19-21].

The purpose of this study was to investigate the feasibility and image quality of ECG-gated DSCTA of the chest as a tool to evaluate cardiac and non-cardiac causes of acute chest pain in patients presenting to the emergency department.

\section{Materials and methods}

\section{Patient population}

Sixty consecutive patients ( 32 females, 28 males, mean age $58.1 \pm 16.3$ years, age range $26-84$ years) were prospectively included in this study. Intake was performed on weekdays from 7 am to $7 \mathrm{pm}$ from August to October 2006. All patients suffered from acute chest pain and were referred to our department to diagnose or to rule out pulmonary embolism $(n=56)$ or aortic dissection $(n=4)$. Inclusion criteria were acute chest pain $>5$ min within the previous $24 \mathrm{~h}$ and/or elevated serum D-dimer levels. Dyspnea and hemodynamic instability were not considered exclusion criteria. Similarly, all patients irrespective of their mean or regularity of heart rate and irrespective of their ability to perform breath-hold were included. Exclusion criteria included pregnancy, previous adverse reaction to iodinated contrast agent, nephropathy (serum creatinine $>1.3 \mathrm{mg} / \mathrm{dl}$ ), elevated troponine-I or creatine kinase-MB level in the initial blood sample, initial diagnostic ECG changes indicating an acute coronary syndrome (i.e., ST elevation or depression $>1 \mathrm{~mm}$, T-wave inversion $>4 \mathrm{~mm}$ in $>2$ anatomically contagious leads), and interference with standard clinical care of patients. The study was approved by the local ethics committee; informed consent was obtained.

\section{Scan protocol and data reconstruction}

All CT examinations were performed on a DSCT scanner (Somatom Definition, Siemens Medical Solutions, Forchheim, Germany). First, a single non-enhanced lowdose scan at the level of the aortic root was obtained. In this slice, a region of interest (ROI) was set in the lumen of the aorta for monitoring intraluminal contrast enhancement. The delay from start of contrast material injection to start of scanning was planned using the bolus-tracking technique. A total of $110 \mathrm{ml}$ iodinated contrast material (iodixanol, Visipaque $320 ; 320 \mathrm{mg} / \mathrm{ml}$, GE Healthcare, Buckinghamshire, UK) was administered at a flow rate of $4 \mathrm{ml} / \mathrm{s}$ via an 18-gauge needle placed into a superficial vein in the left antecubital fossa, followed by $30 \mathrm{ml}$ saline solution at the same flow rate $(4 \mathrm{ml} / \mathrm{s})$. After reaching the preset contrast enhancement level of 80 Hounsfield Units (HU) in the ROI, a breath-hold signal was given and the scan was initiated automatically after a delay of $6 \mathrm{~s}$. A topogram was used for planning the examination and determining the scan range. Data acquisition was performed using a dedicated biphasic chest pain protocol. As illustrated in Fig. 1, the lower chest including the heart was scanned with a tube current time of $320 \mathrm{mAs}$ while the upper chest was scanned with a tube current time product of $160 \mathrm{mAs}$. The borderline between upper and lower scan range was set at approximately $2 \mathrm{~cm}$ below the carina. Data acquisition was performed in a cranio-caudal direction. Acquisition parameters were as follows: detector collimation $2 \times 32 \times 0.6 \mathrm{~mm}$ by using a $\mathrm{z}$-flying focal spot for the simultaneous acquisition of $2 \times 64$ overlapping $0.6-\mathrm{mm}$ slices, gantry rotation time $330 \mathrm{~ms}$, and tube potential $120 \mathrm{kV}$. The pitch varied according to the patient's heart rate and ranged from $0.2-0.43$, with higher pitch at higher heart rates. ECG-pulsing for radiation dose reduction [22] was applied in all patients. At mean heart rates below $60 \mathrm{bpm}$, full tube current was applied

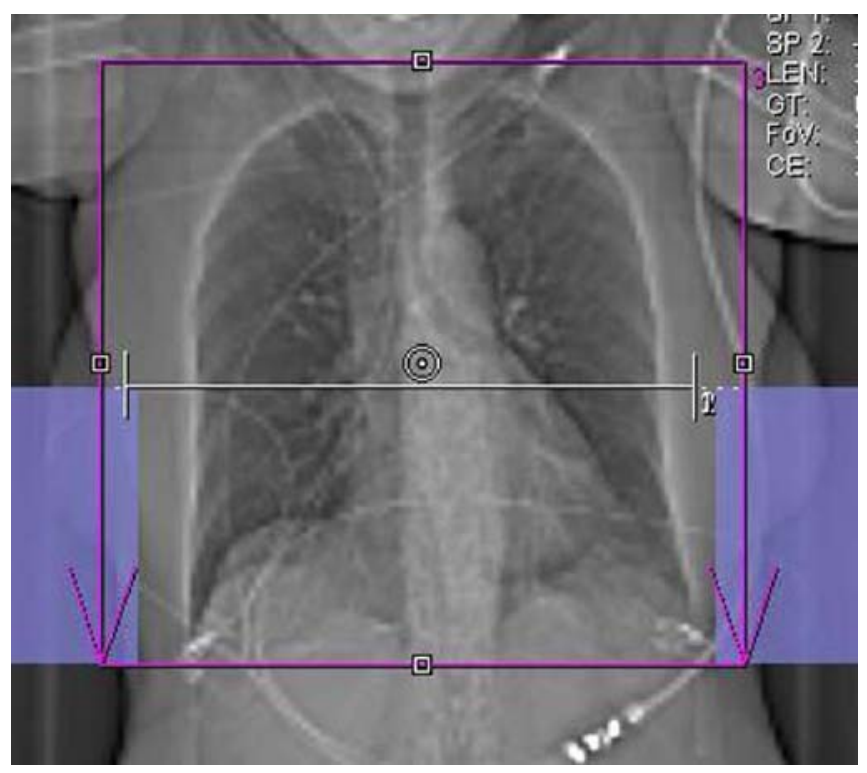

Fig. 1 Scan topogram illustrating planning of the chest pain protocol. The scan range covered the entire chest (red box). Premonitoring for bolus tracking was performed at the level of the aortic root (white line). The border for full tube current for the heart and half tube current for the upper lung is set approximately $2 \mathrm{~cm}$ below the tracheal bifurcation. It is delimited by a virtual horizontal line connecting the upper ends of the blue boxes on both sides 
from 60 to $70 \%$, at $61-70 \mathrm{bpm}$ from 50 to $80 \%$, and at heart rates above 70 from 30 to $80 \%$ of the R-R interval.

Retrospective ECG-gating for phase synchronization was used. For the heart, CT data sets were reconstructed at $70 \%$ of the R-R interval with a slice thickness of $0.75 \mathrm{~mm}$ (increment $0.5 \mathrm{~mm}$ ) by using a medium soft-tissue convolution kernel (B26f) (mean field of view, FoV: $151 \pm 17 \mathrm{~mm}$, image matrix $512 \times 512$ ). If considered necessary, additional images were reconstructed in 5\% steps using the same parameters within the time window of full tube current. Images of the mediastinum (mean FoV: $293 \pm 43 \mathrm{~mm}$ ) including the aorta and pulmonary arteries were reconstructed with a slice thickness of $1 \mathrm{~mm}$ (increment $0.8 \mathrm{~mm}$ ) by using a medium soft-tissue convolution kernel (B30f), and images of the lung were reconstructed with a slice thickness of $2 \mathrm{~mm}$ (increment $1.5 \mathrm{~mm}$ ) by using a sharp convolution kernel (B60f, same FoV as for the mediastinum). All images were transferred to a second Wizard (Siemens) equipped with cardiac post-processing software (Syngo Circulation, Siemens).

\section{Data analysis}

All data were qualitatively evaluated regarding image quality and artifacts of different thoracic structures by two independent readers who are both experienced in cardiovascular radiology. This evaluation was performed on transverse source images, multi-planar reformations (MPR), curved MPR, and thin-slab maximum intensity projections.

Image quality and artifacts-lung parenchyma

Image quality of lung parenchyma was independently rated using a two-point scale, adapted from a previous publication [23]. Lung parenchyma allowing diagnostic assessment due to distinct anatomic details of bronchial and parenchymal structures without significant artifacts and noise was rated with a score of 1 (diagnostic). Lung parenchyma with artifacts or noise causing reduction of image quality and diagnostic value was rated with a score of 2 (non-diagnostic).

Artifacts were rated to quantify the cranio-caudal distribution of artifacts within the lung parenchyma for the right and left lung separately in coronal MPR. Because of the different tube current at upper and lower parts along the $\mathrm{z}$-axis (see Fig. 1), the following scores were separately applied for the apex and the basis of the lung, respectively: $1=$ no artifacts, $2=$ breathing artifacts (stair step artifacts), $3=$ ECG-gating (i.e., synchronization or interpolation artifacts), and $4=$ noise artifacts. If breathing and ECGgating artifacts appeared at the same time in one patient, the artifact with the worst impact on image quality was noted.
Image quality and artifacts-vascular structures

Image quality of thoracic vascular structures was independently rated using the same two-point scale [23] as used for the rating of the lung parenchyma. Regarding the thoracic aorta, a score of 1 (diagnostic) indicated confident evaluation of the ascending aorta, the aortic arch, and descending aorta. Regarding pulmonary arteries, a score of 1 indicated confident evaluation of central, lobar, segmental, or subsegmental pulmonary arteries. Regarding coronary arteries, a score of 1 represented confident depiction (homogenous attenuation; no artifacts decreasing coronary analysis) of the right coronary artery (RCA), left main artery (LMA), left anterior descending artery (LAD), left circumflex artery (LCX), and their side branches. A score of 2 indicated decreased image quality of thoracic vascular structures with severe impairment of diagnostic value (non-diagnostic) due to breathing, motion, or ECG-gating artifacts.

Image noise and attenuation-vascular structures

Image noise was determined as the standard deviation of attenuation in a ROI placed in the ascending aorta [24]. Contrast attenuation was measured in each patient in the ascending aorta, pulmonary trunk, LMA, proximal segment of the RCA, and right and left ventricle. Image noise and contrast attenuation were assessed using a circular ROI positioned exactly within the vessel or ventricular lumen while avoiding superimposition or partial volume effects from the vessel wall or myocardium. Measurements of the ascending aorta and pulmonary trunk as well as of the right and left ventricle were performed on the same transverse image, while the ROI in the coronary arteries was individually placed on separate transverse images.

\section{Imaging findings}

Imaging findings indicating the possible underlying cause of acute chest pain were documented in each patient by both readers in consensus.

\section{Statistical analysis}

Statistical analysis was performed using commercially available software (SPSS 11.5, SPSS Inc., Il). Quantitative variables are expressed as mean \pm standard deviation (SD) including 95\% confidence intervals (CI) or range when appropriate. Categorical data were expressed as frequencies or percentages. Inter-observer agreement (kappa statistics) for image quality ratings was calculated. Twotailed Student's $t$ test for paired samples was used to explore significant differences in vessel attenuation among 


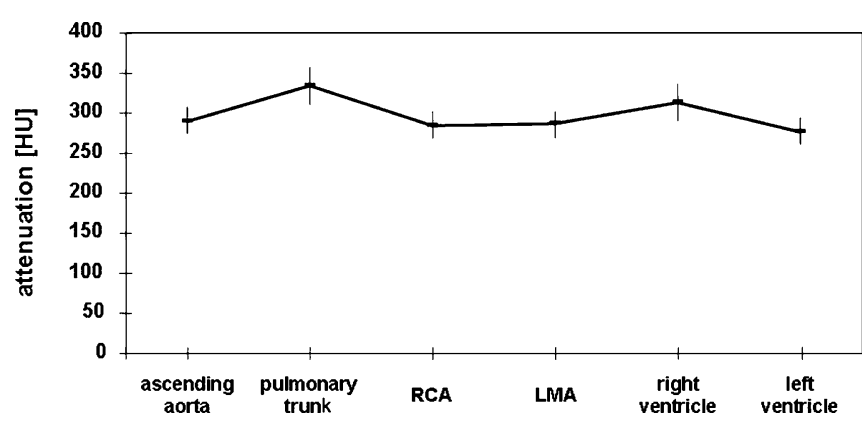

Fig. 2 Mean attenuation values (HU) within different vessels indicating a relatively homogenous contrast distribution between the different thoracic vascular territories. $\mathrm{RCA}=$ right coronary artery; $\mathrm{LMA}=$ left main coronary artery

the ascending aorta, RCA, LMA, pulmonary trunk, and right and left ventricle. Intra-individual differences regarding vessel attenuation among the different vascular territories were performed using pairwise comparisons. Bonferroni correction for multiple comparisons was made, and a $P$-value $<0.003$ was considered statistically significant.

\section{Results}

All CT scans were well tolerated and were successfully performed in all 60 patients without complications. All patients had a sinus rhythm, and the average heart rate during data acquisition was $74.9 \pm 19.0$ beats per minute (bpm) (range $45-130 \mathrm{bpm}$ ). No beta receptor antagonists or benzodiazepines were administered prior to CT; 13 patients $(22 \%)$ took oral beta blockers as part of their baseline medication at the time of the scan. Mean scan time was $12.2 \pm 2.3 \mathrm{~s}$ (range 9.7-17.5 s) and mean scan length was $24.1 \pm 2.1 \mathrm{~cm}$ (range 19.3-29.7 cm). The reconstruction interval used for image reading was $70 \%$ of the R-R interval. In seven patients it was considered necessary to reconstruct additional data sets in 5\% intervals within the window of full tube current to obtain images with diagnostic quality.

Image quality and artifacts-lung parenchyma

Image quality of lung parenchyma was rated as being diagnostic (score 1) by both readers in all 60 patients $(100 \%$; excellent inter-observer agreement, kappa $=1.0)$, and pathology both at upper and lower lung parts could be diagnosed or excluded in all 60 patients.

Lung parenchyma was rated by both readers as being artifact-free in 42 patients $(70 \%)$. Breathing artifacts causing stair-step artifacts were encountered in nine patients $(15 \%)$ by one reader and eight $(13 \%)$ of these nine patients by the other reader (excellent inter-observer agreement, kappa $=0.93$ ). Breathing artifacts were exclusively found at the lung base, i.e., at the end of the breathhold period. ECG-gating-related artifacts were found in ten patients $(17 \%)$ by one reader, and in nine $(15 \%)$ of these ten patients by the other reader (excellent inter-observer

Table 1 Pairwise intra-individual comparisons between different thoracic vascular territories

\begin{tabular}{|c|c|c|c|c|c|}
\hline & & \multirow[t]{2}{*}{ Mean difference } & \multirow[t]{2}{*}{ Standard deviation } & \multicolumn{2}{|c|}{$95 \%$ confidence interval } \\
\hline & & & & Lower boundary & Upper boundary \\
\hline Aorta & Pulmonary trunk & -43 & 11 & -75 & -11 \\
\hline Aorta & RCA & 6 & 5 & -10 & 22 \\
\hline Aorta & LCA & 5 & 4 & -8 & 17 \\
\hline Aorta & Right ventricle & -23 & 13 & -63 & 18 \\
\hline Aorta & Left ventricle & 14 & 5 & -3 & 30 \\
\hline Pulmonary trunk & $\mathrm{RCA}$ & 49 & 11 & 16 & 82 \\
\hline Pulmonary trunk & LCA & 47 & 12 & 12 & 83 \\
\hline Pulmonary trunk & Right ventricle & 20 & 12 & -16 & 57 \\
\hline Pulmonary trunk & Left ventricle & 57 & 9 & 29 & 84 \\
\hline $\mathrm{RCA}$ & LCA & -1 & 6 & -19 & 17 \\
\hline $\mathrm{RCA}$ & Right ventricle & -28 & 13 & -69 & 13 \\
\hline $\mathrm{RCA}$ & Left ventricle & 8 & 5 & -8 & 24 \\
\hline LCA & Right ventricle & -27 & 14 & -70 & 15 \\
\hline LCA & Left ventricle & 9 & 6 & -10 & 28 \\
\hline
\end{tabular}

$\mathrm{RCA}=$ right coronary artery; $\mathrm{LCA}=$ left coronary artery 
Table 2 Imaging findings in the study population

\begin{tabular}{ll}
\hline Diagnosis & Number of patients $(\mathrm{n}=60)$ \\
\hline No pathology & 33 \\
Pulmonary embolism & 11 \\
Aortic pathology, total & 5 \\
Dissection type B & 3 \\
Pseudoaneurysm of the aortic arch & 1 \\
Plaque rupture & 1 \\
Coronary pathology, total & 3 \\
Significant stenosis of the LAD & 2 \\
Occlusion of the RCA & 1 \\
Pulmonary consolidation & 3 \\
Pericarditis/pericardial effusion & 2 \\
Seropneumothorax & 1 \\
Non-small cell lung cancer & 1 \\
Synovial cell carcinoma & 1 \\
\hline
\end{tabular}

$\mathrm{LAD}$, left anterior descending artery; RCA, right coronary artery agreement, kappa $=0.84$ ). The two types of artifacts were not encountered in the same patient, and image quality was diagnostic despite the artifacts.

Image quality and artifacts-vascular structures

Image quality of the thoracic aorta was rated by both readers as being diagnostic (score 1) in all 60 patients $(100 \%$; excellent inter-observer agreement, kappa $=1.0)$, and pathologies of the thoracic aorta could be diagnosed or excluded by both readers in all 60 patients. Both readers rated image quality of the pulmonary arteries in the one (i.e., the same) patient (2\%) as being non-diagnostic (score 2) due to insufficient attenuation of segmental and subsegmental pulmonary arteries. In this patient, attenuation did not allow excluding segmental or subsegmental pulmonary embolism, while attenuation in the pulmonary trunk and right and left lobar artery was considered sufficient for diagnosis or exclusion of central and lobar pulmonary embolism. In this patient, image quality of the coronary
Fig. 3 A 63-year-old female patient admitted to the emergency department with acute chest pain. (a) Curved multiplanar reformations along the centerline of the right coronary (RCA), left anterior descending (LAD), and the left circumflex artery (RCX) allow excluding significant coronary stenosis in this patient. Mean heart rate during DSCTA was $71 \mathrm{bpm}$. (b) Thin-slab transverse maximum intensity projection shows no evidence of pulmonary embolism. (c) Transverse image at the level of the pulmonary trunk demonstrates acute aortic dissection type B (arrow) with mild left-sided pleural effusion
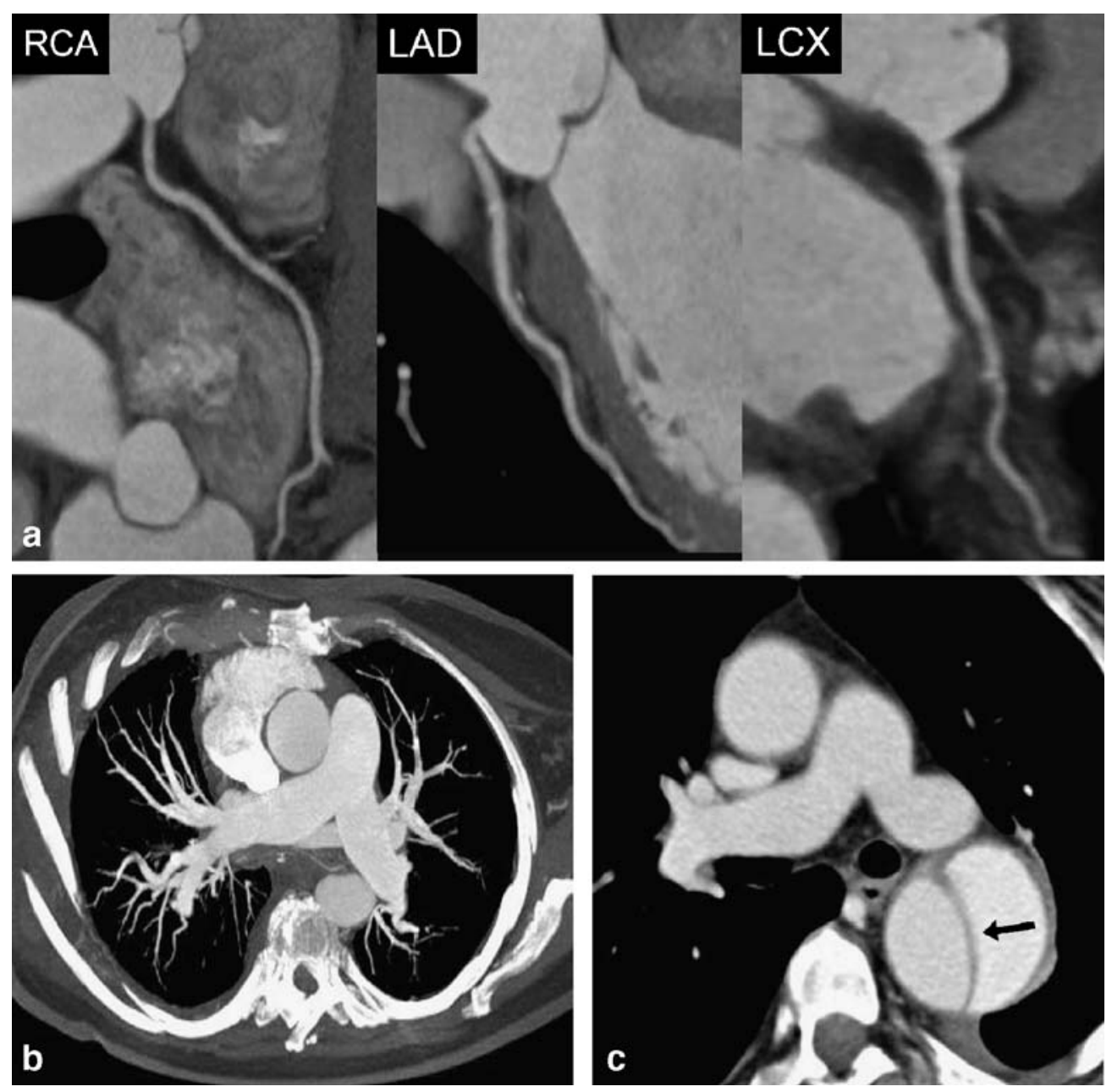
arteries was considered as being diagnostic by both readers. Image quality of coronary arteries was rated by both readers as being diagnostic (score 1) in the same 58 patients (97\%; excellent inter-observer agreement, kappa $=1.0$ ). In the remaining two patients (3\%), image quality of the coronary arteries was considered by both readers as being nondiagnostic (score 2) due to ECG-gating-related artifacts (mean heart rate $71 \mathrm{bpm}$, ranging from 50 to $84 \mathrm{bpm}$ during scanning) in one and severe image noise due to obesity (body mass index $40.4 \mathrm{~kg} / \mathrm{m}^{2}$ ) in the other. In both patients, both readers rated image quality of the pulmonary arteries as being diagnostic.

Image noise and attenuation-vascular structures

Mean image noise in the ascending aorta (mean ROI size $3.6 \pm 0.2 \mathrm{~cm}^{2}$ ) was $29.8 \pm 5.6$.
Mean attenuation in the ascending aorta (same ROI and same ROI size as for measurements of image noise) was $291 \pm 65$ HU (95\% CI: 274-308 HU), mean attenuation in the pulmonary trunk (mean ROI size $2.4 \pm 0.2 \mathrm{~cm}^{2}$ ) was $334 \pm 93 \mathrm{HU}$ (95\% CI: $310-358 \mathrm{HU})$, mean attenuation in the proximal RCA (mean ROI size $0.14 \pm 0.02 \mathrm{~cm}^{2}$ ) was $285 \pm 66$ HU (95\% CI: 268-302 HU), and mean attenuation in the LMA (mean ROI size $0.12 \mathrm{~cm}^{2} \pm 0.01$ ) was $286 \pm 67 \mathrm{HU}$ (95\% CI: 269-304 HU). Mean attenuation in the right ventricle (mean ROI size $2.6 \pm 0.2 \mathrm{~cm}^{2}$ ) was $313 \pm 89 \mathrm{HU}$ (95\% CI: 290-336 HU) and mean attenuation in the left ventricle (mean ROI size $2.6 \pm 0.2 \mathrm{~cm}^{2}$ ) was $277 \pm 66 \mathrm{HU}$ (95\% CI: 260-294 HU) (Fig. 2).

No significant differences regarding attenuation were found between the RCA and LMA ( $P=$ n.s.), and between the ascending aorta and both coronary arteries $(P=$ n.s. $)$. No significant differences in attenuation were present between the right and left ventricle $(P=$ n.s. $)$, between the right ventricle and ascending aorta $(P=$ n.s. $)$, between the right

Fig. 4 A 58-year-old female patient admitted to the emergency department with acute chest pain. (a) Curved multiplanar reformations along the centerline of the right coronary (RCA), left anterior descending (LAD), and the left circumflex artery (LCX) demonstrate normal coronary arteries and no evidence of stenosis. Mean heart rate during DSCTA was $63 \mathrm{bpm}$. (b) Thin-slab transverse maximum intensity projection show bilateral pulmonary embolism (arrows). (c) Oblique-sagittal thin-slab maximum intensity projection demonstrates the thoracic aorta without evidence of disease
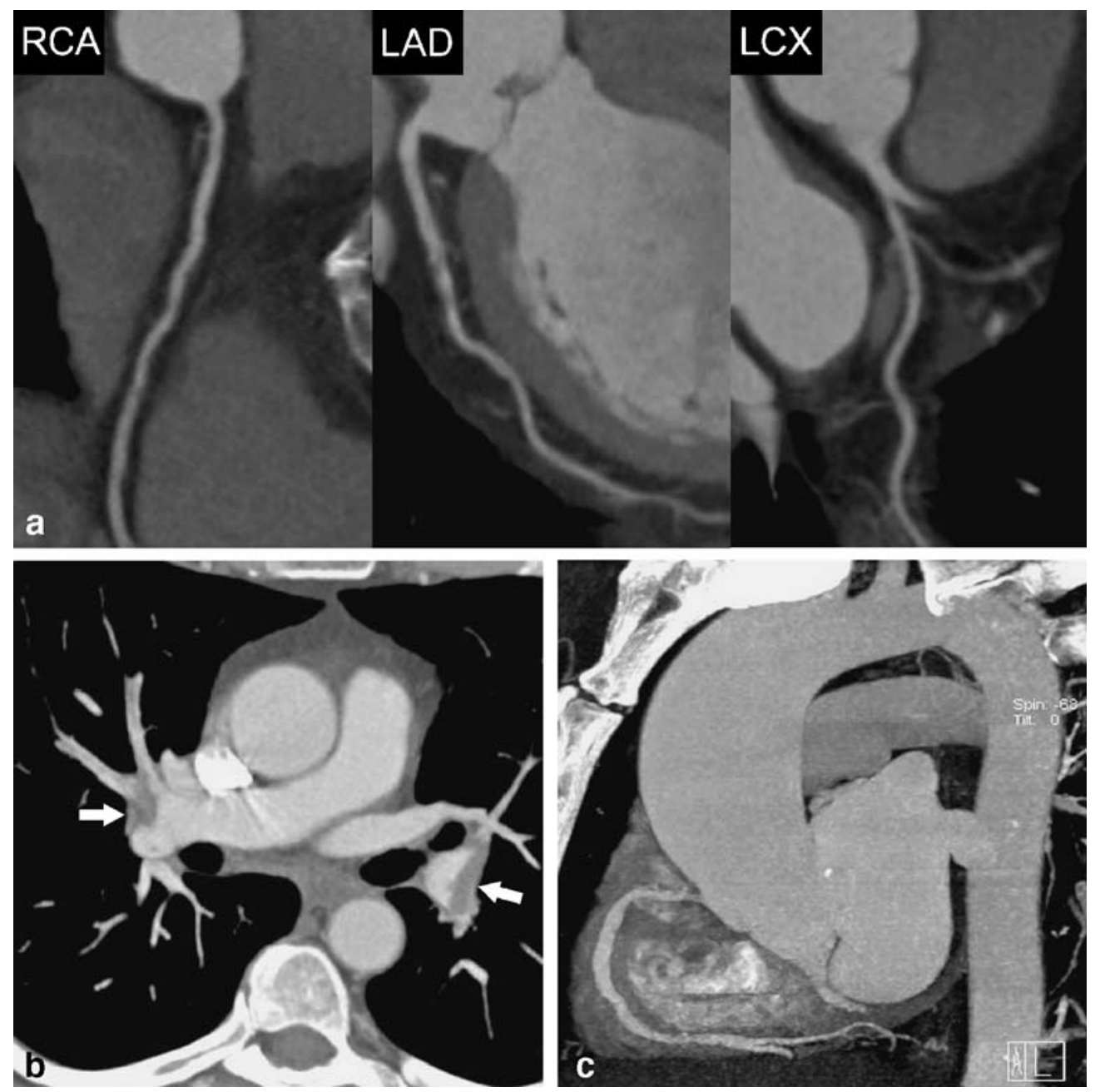
ventricle and pulmonary trunk $(P=$ n.s. $)$, and between the right ventricle and the RCA ( $P=$ n.s. $)$ and LMA $(P=$ n.s. $)$. Similarly, there were no significant differences in attenuation between the left ventricle and ascending aorta $(P=$ n.s. $)$, and between left ventricle and LMA ( $P=$ n.s. $)$ and RCA $(P=$ n.s.). Significant differences were only found between the pulmonary trunk and ascending aorta $(\mathrm{P}<0.003)$, between the pulmonary trunk and the RCA and LMA $(P<0.003)$, respectively, and between the left ventricle and pulmonary trunk $(P<0.003)$.

Pairwise comparisons of mean intra-individual differences regarding vessel attenuation were small and ranged between $1.18 \pm 5.86 \mathrm{HU}$ comparing the right and left coronary artery and $56.45 \pm 8.96 \mathrm{HU}$ comparing the pulmonary trunk and left ventricle (Table 1).

\section{Imaging findings}

No pathologic findings were found in 33 patients $(55 \%)$, whereas various pathologies of the aorta, pulmonary and coronary arteries, lung, and mediastinum were present in 27 patients (45\%) (Table 2, Figs. 3, 4, 5).

\section{Discussion}

Our study demonstrates that DSCT allows performing an ECG-gated chest examination for visualizing the different thoracic vascular territories at the same time-by employing an adjusted contrast media protocol-within a reasonable breath-hold period, and thus provides a diagnostic image quality in almost all patients. One of the most important findings of this study is that diagnostic data of coronary arteries could be obtained without foregoing heart rate control.

\section{Contrast media protocol and scan time}

With increasing gantry rotation times and faster volume coverage of newer CT scanners, scan times successively

Fig. 5 A 71-year-old male patient admitted to the emergency department with acute chest pain. (a) Curved multiplanar reformations along the centerline of the right coronary (RCA), left anterior descending (LAD), and the left circumflex artery (LCX) show occlusion of the proximal RCA (long arrow) and vessel wall calcifications without significant stenosis in the proximal and middle segment of the LAD and LCX (short arrows). Mean heart rate during DSCTA was $73 \mathrm{bpm}$.

(b) Thin-slab transverse maximum intensity projection demonstrates normal opacification of pulmonary arteries with no evidence of embolism. (c) Oblique-sagittal thin-slab maximum intensity projection demonstrates the thoracic aorta with minimal atherosclerotic wall changes, but with no evidence of potential causes for acute chest pain
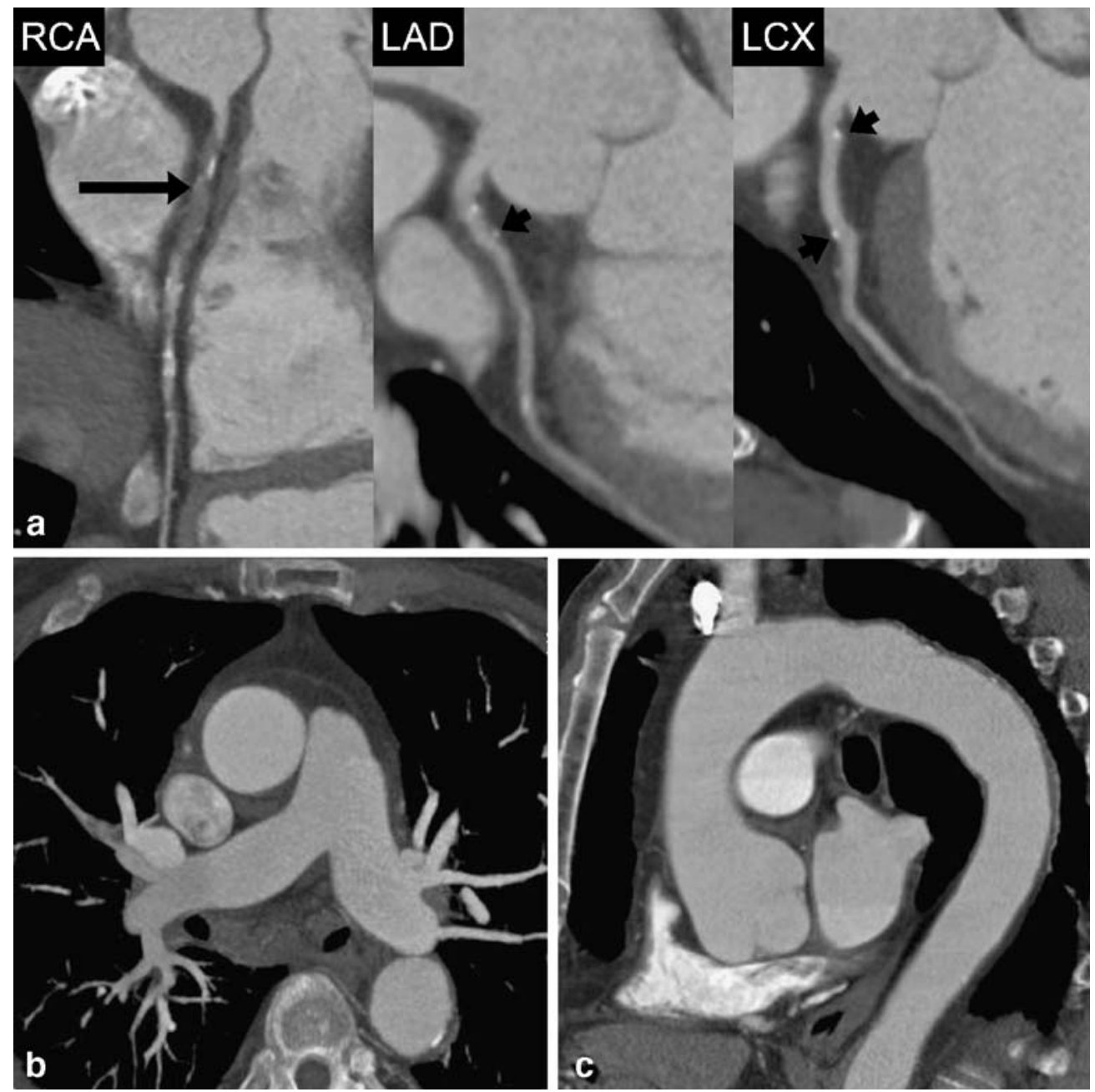
shorten and higher injection rates are required to achieve sufficient contrast in the vascular territory of interest. Similar to a previous 64-slice CT study [15], we have set the ROI for bolus tracking in the ascending aorta. With a relatively low threshold of $80 \mathrm{HU}$ above which the scan was initiated, a homogenous attenuation in both aortic/ coronary and pulmonary arteries could be achieved. By doing so, we aimed at the time interval where the contrast enhancement curve of the pulmonary circulation overlaps with enhancement of the aorta [25]. This has also resulted in comparable attenuation in left and right cardiac cavities. Recommended mean attenuation values for diagnostic image quality are approximately $300 \mathrm{HU}$ for the pulmonary arteries [26] and 250-350 HU in the ascending aorta and
Fig. 6 Flow chart of a generally accepted clinical pathway for patients with cardiac and noncardiac causes of acute chest pain. CT represents the standard of reference in patients with suspicion of acute aortic syndromes or pulmonary embolism (light gray boxes). The added value of CT in the evaluation of acute coronary syndrome has been already demonstrated [8] (moderate gray box). The potential future role of CT in patients with acute chest pain might be at an even earlier point of diagnostic work-up (dark gray boxes) to rule out lifethreatening coronary, pulmonary, and aortic disease and to guide adequate therapeutic interventions

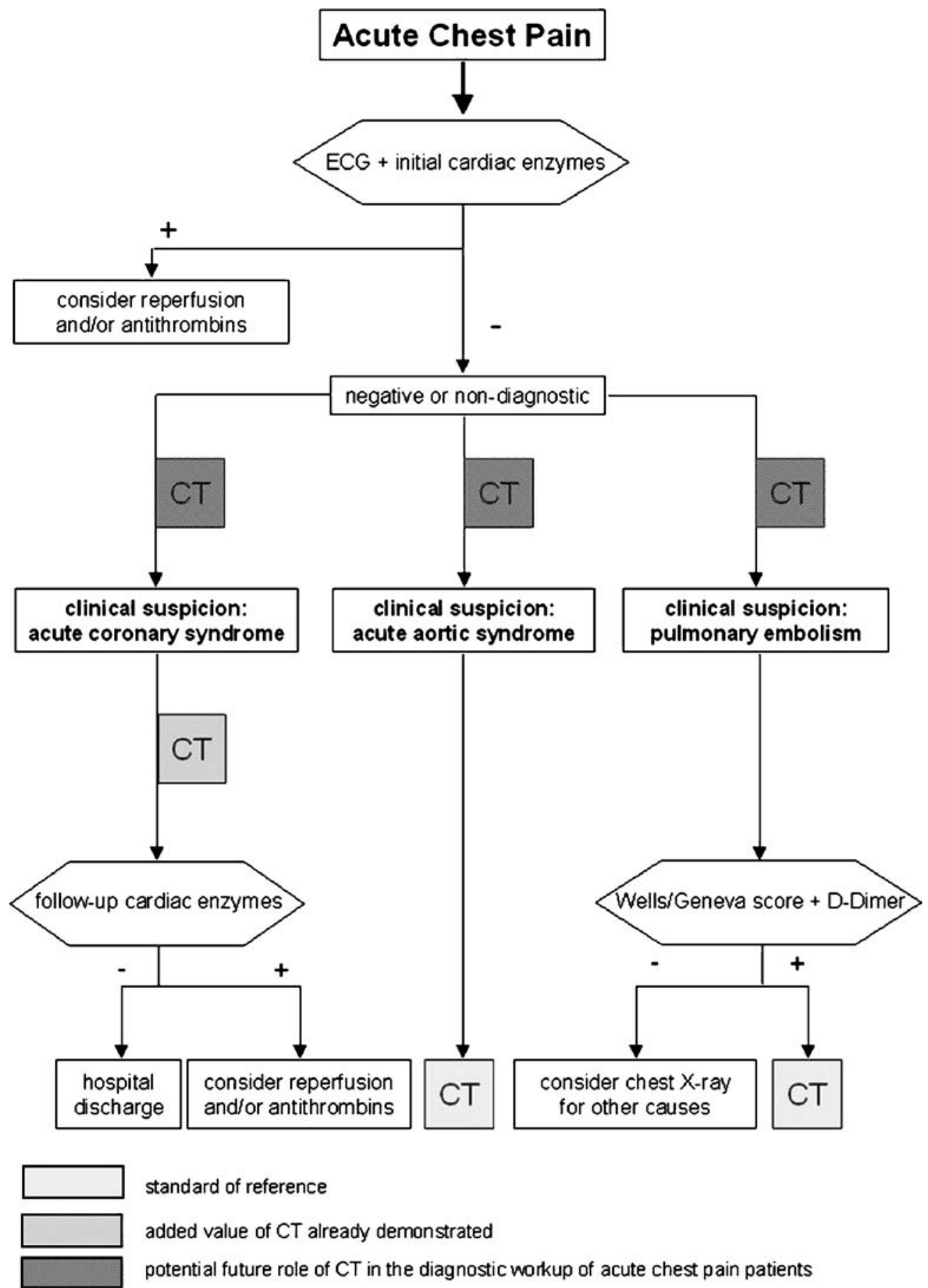


coronary arteries $[27,28]$. We obtained a mean attenuation of $291 \mathrm{HU}$ in the ascending aorta, $334 \mathrm{HU}$ in the pulmonary trunk, $285 \mathrm{HU}$ in the RCA, and $286 \mathrm{HU}$ in the LMA with only small intra- and inter-individual variations. These attenuation values were considered diagnostic in $98 \%$ of our patients and are comparable to previously published data $[15,29]$. We encountered only one patient with non-diagnostic attenuation of segmental and subsegmental pulmonary arteries. However, this may have been also caused by other reasons negatively affecting contrast attenuation in pulmonary arteries such as Valsalva maneuver [30].

The other major issue for CT in acute chest pain represents the issue of scan duration. As ECG-gated CT requires low pitch [31], scan time is increased as compared to non-gated chest CT examinations. This, however, negatively affects image quality by making examinations more prone to breathing artifacts. This holds particularly true for patients with acute chest pain who often suffer from dyspnea. In our study, mean scan time was approximately $12 \mathrm{~s}$, which represents a further improvement as compared to a mean scan time of $21 \mathrm{~s}$ with 64-slice CT [15].

Role of CT in the diagnostic pathway of acute chest pain

Figure 6 represents a simplified flow-chart of a generally accepted clinical pathway for patients with cardiac and non-cardiac causes of acute chest pain [9,32,33]. While $\mathrm{CT}$ is the accepted reference modality for the diagnosis and exclusion of pulmonary embolism and aortic dissection $[10,11]$, the role of $\mathrm{CT}$ in patients with a suspected cardiac cause for chest pain is currently under investigation. Gallagher and colleagues [6] have shown that 64slice CT has accuracy that is comparable to that of stress nuclear imaging for the detection of acute coronary syndrome in low-risk patients with negative serial ECG and biomarker results. Hoffmann and coworkers [8] have demonstrated that 64-slice CT has good performance characteristics for ruling out acute coronary syndromes in patients presenting to the emergency department with acute chest pain. The authors demonstrated in patients in whom initial triage was inconclusive that the absence of coronary artery plaque or significant stenosis on CT angiography had an excellent negative predictive value for the subsequent diagnosis of acute coronary syndrome. Furthermore, in those patients with CAD on CT, the extent of coronary atherosclerotic plaque provided incremental information to standard baseline patient variables and clinical risk assessment. Goldstein and colleagues [7] have shown that 64-slice CT coronary angiography is able to definitely establish or exclude CAD as the cause of acute chest pain. The high negative predictive value of the studies suggests that CT coronary angiography may be useful for facilitating and optimizing triage of patients with acute chest pain and/or inconclusive initial emergency department evaluation $[7,8]$.

\section{Study limitations}

We included only a relatively small number of patients and thus a small number of pathologies. Second, our study lacks a standard end-point to assess the final diagnosis and misses comparison with the reference standard invasive coronary angiography for investigating $\mathrm{CAD}$ for which diagnostic accuracy could be calculated. However, only a minority of our patients underwent invasive coronary angiography because in our emergency department standard management in patients with suspected acute coronary syndrome directly undergoing invasive coronary angiography without prior CT. That is in line with most studies involving patients referred to the emergency department [34]. Third, we did not investigate the optimal contrast media technique for obtaining homogenous contrast attenuation of different thoracic vascular territories. It remains to be determined if the use of the test bolus technique would yield better results with regard to vessel opacification. Finally, it needs to be assessed whether caudo-cranial or caudo-cranial scan direction will result in better image quality.

\section{Conclusion}

First experience indicates that DSCTA is feasible in patients with acute chest pain and provides diagnostic image quality of the aorta, pulmonary arteries, and coronary artery system as well as of the lung parenchyma and mediastinum in a patient population without foregoing heart rate control. A dedicated contrast media protocol allows for homogenous attenuation of the different thoracic vascular territories.

Acknowledgement This study has been supported by the National Center of Competence in Research, Computer-Aided and ImageGuided Medical Interventions of the Swiss National Science Foundation. 


\section{References}

1. Swap CJ, Nagurney JT (2005) Value and limitations of chest pain history in the evaluation of patients with suspected acute coronary syndromes. Jama 294:2623-2629

2. Zimmerman J et al (1999) Diagnostic marker cooperative study for the diagnosis of myocardial infarction. Circulation 99:1671-1677

3. Fesmire FM et al (2002) The Erlanger chest pain evaluation protocol: a oneyear experience with serial 12-lead ECG monitoring, two-hour delta serum marker measurements, and selective nuclear stress testing to identify and exclude acute coronary syndromes. Ann Emerg Med 40:584-594

4. Hoffmann U et al (2006) MDCT in early triage of patients with acute chest pain. AJR Am J Roentgenol 187: 1240-1247

5. Sato Y et al (2005) Efficacy of multislice computed tomography for the detection of acute coronary syndrome in the emergency department. Circ J 69:1047-1051

6. Gallagher MJ, Ross MA, Raff GL, Goldstein JA, O’Neill WW, O’Neil B (2007) The diagnostic accuracy of 64-slice computed tomography coronary angiography compared with stress nuclear imaging in emergency department low-risk chest pain patients. Ann Emerg Med 49:125-136

7. Goldstein JA, Gallagher MJ, O’Neill WW, Ross MA, O’Neil B, Raff GL (2007) A randomized controlled trial of multi-slice coronary computed tomography for evaluation of acute chest pain. J Am Coll Cardiol 49:863-871

8. Hoffmann U et al (2006) Coronary multidetector computed tomography in the assessment of patients with acute chest pain. Circulation 114:2251-2260

9. Erhardt L et al (2002) Task force on the management of chest pain. Eur Heart J 23:1153-1176

10. Quiroz R et al (2005) Clinical validity of a negative computed tomography scan in patients with suspected pulmonary embolism: a systematic review. Jama 293:2012-2017
11. Hayter RG, Rhea JT, Small A, Tafazoli FS, Novelline RA (2006) Suspected aortic dissection and other aortic disorders: multi-detector row CT in 373 cases in the emergency setting. Radiology 238:841-852

12. Leschka S et al (2005) Accuracy of MSCT coronary angiography with 64-slice technology: first experience. Eur Heart J 26:1482-1487

13. Raff GL, Gallagher MJ, O'Neill WW, Goldstein JA (2005) Diagnostic accuracy of noninvasive coronary angiography using 64-slice spiral computed tomography. J Am Coll Cardiol 46:552-557

14. Schertler T, Glucker T, Wildermuth S, Jungius KP, Marincek B, Boehm T (2005) Comparison of retrospectively ECG-gated and nongated MDCT of the chest in an emergency setting regarding workflow, image quality, and diagnostic certainty. Emerg Radiol 12:19-29

15. Johnson TR et al (2007) ECG-gated 64-MDCT angiography in the differential diagnosis of acute chest pain. AJR Am J Roentgenol 188:76-82

16. White C, Read K, Kuo D (2006) Assessment of chest pain in the emergency room: what is the role of multidetector CT? Eur J Radiol 57:368-372

17. Mollet NR et al (2005) High-resolution spiral computed tomography coronary angiography in patients referred for diagnostic conventional coronary angiography. Circulation 112:2318-2323

18. Flohr TG et al (2006) First performance evaluation of a dual-source CT (DSCT) system. Eur Radiol 16:256-268

19. Achenbach S et al (2006) Contrastenhanced coronary artery visualization by dual-source computed tomographyinitial experience. Eur J Radiol 57:331335

20. Johnson TR et al (2006) Dual-source CT cardiac imaging: initial experience. Eur Radiol 16:1409-1415

21. Scheffel H et al (2006) Accuracy of dual-source CT coronary angiography: First experience in a high pre-test probability population without heart rate control. Eur Radiol 16:2739-2747

22. Jakobs TF et al (2002) Multislice helical CT of the heart with retrospective ECG gating: reduction of radiation exposure by ECG-controlled tube current modulation. Eur Radiol 12:1081-1086
23. d'Agostino AG et al (2006) Low-dose ECG-gated 64-slices helical CT angiography of the chest: evaluation of image quality in 105 patients. Eur Radiol 16:2137-2146

24. Lembcke A et al (2004) Image quality of noninvasive coronary angiography using multislice spiral computed tomography and electron-beam computed tomography: intraindividual comparison in an animal model. Invest Radiol 39:357-364

25. Lee CH et al (2007) Determination of optimal timing window for pulmonary artery MDCT angiography. AJR Am J Roentgenol 188:313-317

26. Prokop M (2000) Multislice CT angiography. Eur J Radiol 36:86-96

27. Becker CR et al (2003) Optimal contrast application for cardiac 4-detectorrow computed tomography. Invest Radiol 38:690-694

28. Husmann L et al (2006) Influence of cardiac hemodynamic parameters on coronary artery opacification with 64-slice computed tomography. Eur Radiol 16:1111-1116

29. Raptopoulos VD et al (2006) MDCT angiography of acute chest pain: evaluation of ECG-gated and nongated techniques. AJR Am J Roentgenol 186: S346-S356

30. Wittram C (2003) Pulmonary artery enhancement at CT pulmonary angiography. Radiology 229:932; author reply $932-933$

31. Primak AN, McCollough CH, Bruesewitz MR, Zhang J, Fletcher JG (2006) Relationship between noise, dose, and pitch in cardiac multidetector row CT. Radiographics 26:1785-1794

32. Lee TH, Goldman L (2000) Evaluation of the patient with acute chest pain. N Engl J Med 342:1187-1195

33. van Belle A et al (2006) Effectiveness of managing suspected pulmonary embolism using an algorithm combining clinical probability, D-dimer testing, and computed tomography. Jama 295:172-179

34. Ioannidis JP, Salem D, Chew PW, Lau J (2001) Accuracy of imaging technologies in the diagnosis of acute cardiac ischemia in the emergency department: a meta-analysis. Ann Emerg Med $37: 471-477$ 